Article

\title{
Comparison of Superior Mesenteric Artery Remodeling and Clinical Outcomes between Conservative or Endovascular Treatment in Spontaneous Isolated Superior Mesenteric Artery Dissection
}

\author{
Sz-Han $\mathrm{Yu}^{1}$, Ing-Heng Hii ${ }^{2}$ and I-Hui $\mathrm{Wu}^{1,3, * \mathbb{D}}$ \\ 1 Department of Surgery, Cardiovascular Section, National Taiwan University Hospital, Taipei 10002, Taiwan; \\ a0915957593@gmail.com \\ 2 Department of Surgery, Cardiovascular Section, Dalin Tzu Chi Hospital, Chaiyi 62247, Taiwan; \\ heng_1014@hotmail.com \\ 3 Graduate Institute of Clinical Medicine, College of Medicine, National Taiwan University, \\ Taipei 10002, Taiwan \\ * Correspondence: aaronihuiwu@gmail.com; Tel.: +886-2-23123456 (ext. 65735); Fax: +886-2-23956934
}

check for

updates

Citation: Yu, S.-H.; Hii, I.-H.;

Wu, I.-H. Comparison of Superior

Mesenteric Artery Remodeling and

Clinical Outcomes between

Conservative or Endovascular

Treatment in Spontaneous Isolated

Superior Mesenteric Artery

Dissection. J. Clin. Med. 2022, 11, 465.

https://doi.org/10.3390/jcm11020465

Academic Editor:

Roland Klingenberg

Received: 9 November 2021

Accepted: 13 January 2022

Published: 17 January 2022

Publisher's Note: MDPI stays neutral with regard to jurisdictional claims in published maps and institutional affiliations.

Copyright: (C) 2022 by the authors. Licensee MDPI, Basel, Switzerland. This article is an open access article distributed under the terms and conditions of the Creative Commons Attribution (CC BY) license (https:// creativecommons.org/licenses/by/ $4.0 /)$.

\begin{abstract}
The aim of this study was to analyze the superior mesenteric artery (SMA) remodeling after initial conservative or endovascular treatment with a standardized definition and midterm outcomes in patients with spontaneous isolated dissection of the superior mesenteric artery (SIDSMA). This retrospective study enrolled patients with SIDSMA from January 2007 to August 2019. All patients were treated initially with conservative treatment. If they failed the medical treatment, they were converted to interventional treatment. The morphological endpoint was determined by the standardized SMA remodeling, and the clinical endpoints were determined by the in-hospital mortality, hospital stay, and the bowel-related mid-term mortality. A total of 34 consecutive patients with SIDSMA were identified. Twenty-three $(67.6 \%)$ and eleven $(33.4 \%)$ patients underwent conservative and interventional treatments, respectively. Clinical features and morphologic changes on CTA were available in $25(73.5 \%)$ patients during the median follow-up of 23.3 months. Standardized SMA remodeling was significantly $(p<0.05)$ better in patients undergoing endovascular stenting, especially in patients with Yun's IIb classification. There was no mesenteric ischemia or SMA aneurysm during follow-up period. Patients with SIDSMA can be treated safely with initial conservative treatment. However, significant portions of patients will require endovascular intervention due to the persistent symptoms. Clinically endovascular stenting could be performed successfully, and SMA remodeling was satisfactory during the mid-term follow-up.
\end{abstract}

Keywords: dissection; mesenteric artery; treatment outcome; endovascular stent

\section{Introduction}

Spontaneous isolated dissection of the superior mesenteric artery (SIDSMA) is rare but increasingly diagnosed because of the widespread availability of high-quality computed tomography angiography (CTA), especially in the lack of specific lab tests and clinical signs. The etiology of SIDSMA remains unclear. Hypertension, genetic variants, and the mechanical stress at the anterior wall of the SMA near the convex curvature have all been proposed [1]. Previous reports [2,3] have indicated that risk factors of SIDSMA included male, smoking, middle-aged, hypertension, and Asian population [1-8]. Clinical presentations can range from incidental CTA finding without symptoms to acute abdominal pain and can progress to superior mesenteric artery (SMA) occlusion and mesenteric ischemia. The CTA morphology alone could not predict the clinical course $[3,9,10]$. Initial treatment can always be managed conservatively with bowel rest, hydration, blood pressure 
control, and analgesia, with or without anticoagulation. In asymptomatic patients, conservative treatment is safe, and none of these patients required secondary intervention [11]. In symptomatic patients, the symptom relief rate ranges from $66.8 \%$ to $86.6 \%$ after one week $[1,12,13]$. However, $5.0-44.0 \%$ percent of patients still fail the medical treatment or require conversion to interventional therapy, predominately endovascular SMA stent, with a small percentage (1.0-3.0\%) receiving open revascularization if progressive mesenteric ischemia led to bowel resection [9,14]. Given the dissected nature, one might also expect the aneurysmal degeneration of SMA in the following years, resulting in recurrent symptoms, aneurysm rupture, or bowel ischemia. In order to determine the optimal treatment, positive SMA remodeling is paramount. In the current literature, there was no standardized definition and quantitative comparison for the positive SMA remodeling based on the CTA morphological change over the follow-up between the interventional and medical treatments [5,14-17]. The use of antiplatelet or anticoagulation therapy to prevent SMA thrombosis and promote positive SMA remodeling is also controversial $[9,11,16]$.

In this study, we investigated the risk factors of failure of initial medical therapy, the comparison of SMA remodeling between the intervention and conservative treatments by the CTA morphological change in the mid-term follow-up, and the clinical outcomes by reviewing patients with SIDSMA undergoing initial conservative treatment.

\section{Materials and Methods}

\subsection{Study Patients}

This retrospective study included 34 consecutive patients with SIDSMA at the National Taiwan University Hospital between January 2007 and August 2019. The follow-up data were truncated in March 2020. This study was approved by the Institutional Review Board of National Taiwan University Hospital (NTUH-202104075RINA). An informed consent was waived for this retrospective review.

The diagnosis of SIDSMA was made from findings on the axial views of CTA with an intimal flap in the SMA with/without false lumen (FL) thrombosis (Figure 1). Patients with concomitant aortic and SMA dissection were excluded from this study. Presenting symptoms included abdominal pain, nausea, and/or vomiting. The pain severity score was measured on a 10-point visual analog scale, and the duration of abdominal pain was also investigated. The morphological CTA findings adopted the Yun's classification [3]. It classified the dissection of the SMA at CTA as type I (entry and re-entry tear visible, patent FL), type IIa (only entry tear visible, patent FL), type IIb (FL thrombosis, patent true lumen (TL), and type III (TL and FL occlusion). TL and FL diameters as well as the length of SMA dissection were recorded. Data collected on all patients included age, sex, comorbidities, medical histories, follow-up duration, length of hospital stay, change of symptoms, and treatment strategies. Morphology of the SMA dissection and morphological changes in the CTA follow-up were analyzed and compared between conservative and endovascular SMA stent (EVT) groups. 


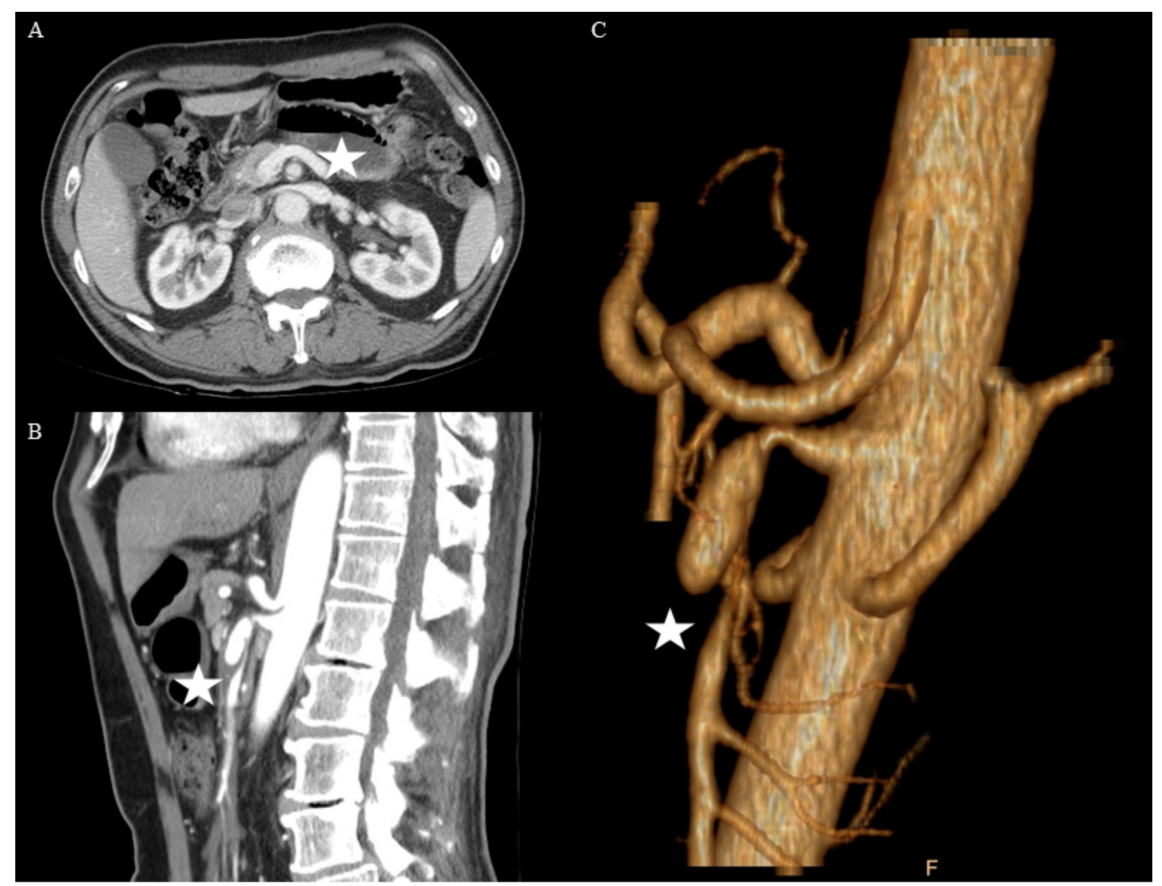

Figure 1. This was a 66-year-old man with Yun's Ila spontaneous isolated dissection of the superior mesenteric artery (asterisks). Computed tomography (CT) scans, (A) axial, (B) sagittal, and (C) threedimensional, demonstrated small patent true lumen, and conservative treatment was applied first.

\subsection{Treatment Algorithm}

The asymptomatic SIDSMA patients were detected incidentally during the abdominal workup. They all received conservative treatment with the preexisting medications and imaging surveillance at outpatient department. The symptomatic group was hospitalized in all cases, and they all received initial conservative treatment, which included food withdrawal, hydration, analgesia, and blood pressure control with the anti-impulse therapy first. Pain resolution was recognized as no more analgesics before or after oral intake. In selecting the antithrombotic agent, the presence of cardiovascular disease and the use of any antiplatelet agent before the onset of SIDSMA were considered. No additional antithrombotic agents were required if there were no pre-existing comorbidities. If a patient presented with suspicious bowel ischemia symptoms, persisted abdominal pain for more than two days, or progressive SMA dissection on CTA, catheter-based SMA stenting was performed with the 0.018 wire system via either the brachial or femoral approach with the self-expandable bare metal stents. The distal stent landing zone was chosen based on the angiography and was usually one centimeter beyond the distal end of SMA dissection. The size of the SMA stent was chosen based on the total diameter of dissected SMA distally and proximally. The size of the stent was usually $5 \mathrm{~mm}$ in the distal end, and telescoped to $8 \mathrm{~mm}$ proximally. The length of the stent was measured from the end of distal SMA dissection and extended 3-5 mm beyond the orifice of the SMA into the aorta. No distal intimal tear was ever noted. The antithrombotic agents were added with mono or dual antiplatelet agents. Open surgery was considered in patients after failed endovascular intervention and bowel infarction (Figure 2). 


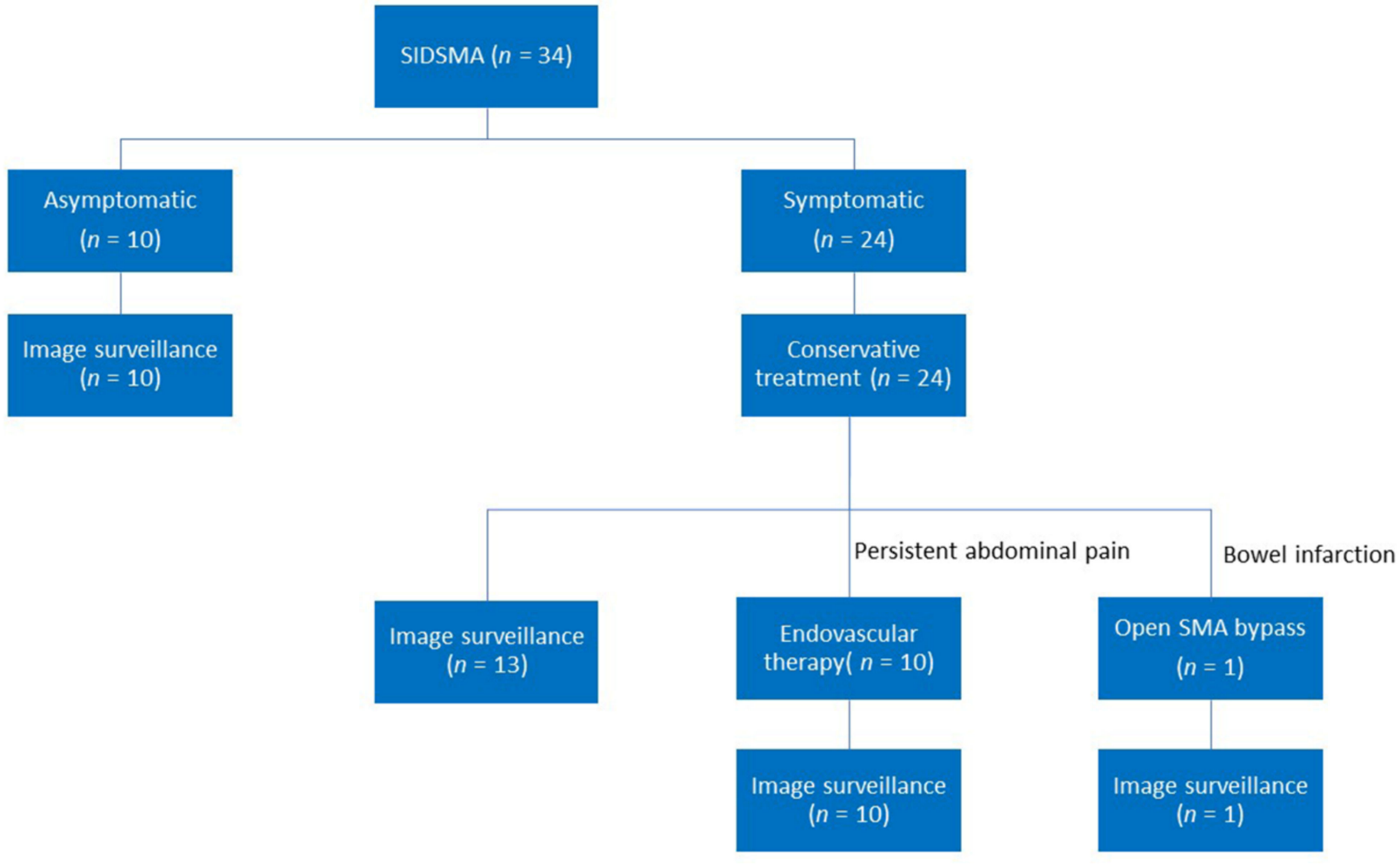

Figure 2. Patient treatment algorithm. SIDSMA: spontaneous isolated dissection of superior mesenteric artery, SMA: superior mesenteric artery.

\subsection{SMA Remodeling and Outcome Measurement}

Positive SMA remodeling was defined as the FL reduction, TL expansion with no growth, or reduction in total SMA diameter during the follow-up. Once patients discharged after conservative or intervention treatment, they were periodically followed-up at an outpatient clinic to assess the recurrence of abdominal symptoms and morphologic changes of the SMA lesion at 3- and 6-month intervals and annually. SMA aneurysm was defined as more than $2.0 \mathrm{~cm}$ in diameter [14]. If no symptom recurrence or interval changes on CT images occurred after 1 year, CTA was performed biannually. In this study, SMA morphological comparison was only performed between EVT and conservative treatment.

\subsection{Statistical Analysis}

Binary variables were recorded as a number and percentage. Continuous variables were assessed as mean \pm standard deviation or median with interquartile range as appropriate, and analysis was completed with student $t$-test and Mann-Whitney test. Potential risk factors were examined by simple and multiple linear regression with beta coefficient presented. The change in TL and FL diameters before and after intervention were graphed and analyzed by paired $t$-test and Wilcoxon Signed-rank test. Statistics were performed with SAS 9.4 software (SAS Inc., Cary, NC, USA), and $p$-value $<0.05$ was considered statistically significant.

\section{Results}

During the study period from January 2007 to August 2019, a total of 34 consecutive patients with SIDSMA were identified (median age: 58 years, range: 47.3-64.8; male, $n=31$; $91.2 \%$ : symptomatic, $n=24 ; 70.6 \%$ ) at a single institution.

\subsection{Patient Characteristics}

Clinical characteristics are summarized in Table 1 . Hypertension $(n=17,50.0 \%)$ was the most common risk factor. Abdominal pain $(n=24,70.6 \%)$ was the most prominent 
symptom in SIDSMA and accounted for all symptomatic patients. In these 24 symptomatic patients, the median abdominal pain severity score was 6.0 , and all these patients received initial conservative treatment. Twenty-three (67.6\%) patients underwent primary conservative, and $11(33.4 \%)$ patients underwent interventional treatments, respectively. The median time of abdominal pain was significantly longer in the interventional group compared to the medical treatment group. The median length of SMA dissection was $65.8 \mathrm{~mm}$, range: 38.3-107.3. The patients were classified into four types according to Yun's classification [6]: type I ( $n=4,11.8 \%)$, IIa $(n=5,14.7 \%), \operatorname{Ilb}(n=23,67.6 \%)$, and III $(n=2,5.9 \%)$. No significant differences were observed between the angiographic types regarding pain severity and duration at initial presentation. Clinical characteristics categorized by symptomatic or asymptomatic SIDSMA are illustrated in Supplementary Table S1.

Table 1. Patient demographics, CT scan morphology, and follow-up data.

\begin{tabular}{|c|c|c|c|c|c|c|c|}
\hline \multirow[b]{3}{*}{ Age } & \multirow{2}{*}{\multicolumn{2}{|c|}{$\begin{array}{c}\text { Total } \\
n=34\end{array}$}} & \multirow{2}{*}{\multicolumn{2}{|c|}{$\begin{array}{c}\text { Conservative } \\
n=23\end{array}$}} & \multirow{2}{*}{\multicolumn{2}{|c|}{$\begin{array}{c}\text { EVT } \\
n=11\end{array}$}} & \multirow{3}{*}{$\begin{array}{c}\boldsymbol{p} \text {-Value } \\
0.392\end{array}$} \\
\hline & & & & & & & \\
\hline & 58.0 & $47.3-64.8$ & 58.0 & $46.0-65.0$ & 58.0 & $51.0-62.0$ & \\
\hline Male & 31.0 & $91.2 \%$ & 21.0 & $91.3 \%$ & 10.0 & $90.9 \%$ & 0.704 \\
\hline BMI & 25.3 & $22.4-27.2$ & 26.0 & $21.7-27.9$ & 24.6 & $22.8-26.1$ & 0.274 \\
\hline Smoking & 9.0 & $26.5 \%$ & 6.0 & $26.1 \%$ & 3.0 & $27.3 \%$ & 0.625 \\
\hline Alcohol & 5.0 & $14.7 \%$ & 3.0 & $13.0 \%$ & 2.0 & $18.2 \%$ & 0.529 \\
\hline Hypertension & 17.0 & $50.0 \%$ & 12.0 & $52.2 \%$ & 5.0 & $45.5 \%$ & 0.362 \\
\hline $\mathrm{SBP}(\mathrm{mm} \mathrm{Hg})$ & 143.0 & $135.0-164.0$ & 143.0 & $137-164$ & 141.0 & 135.0 & 0.202 \\
\hline DBP (mm Hg) & 90.0 & $83.0-97.0$ & 90.0 & $82-95$ & 91.0 & 83.0 & 0.388 \\
\hline $\mathrm{DM}$ & 4.0 & $11.8 \%$ & 2.0 & $8.7 \%$ & 2.0 & $18.2 \%$ & 0.389 \\
\hline Dyslipidemia & 3.0 & $8.8 \%$ & 3.0 & $13.0 \%$ & 0.0 & $0.0 \%$ & 0.296 \\
\hline CAD & 1.0 & $2.9 \%$ & 0.0 & $0.0 \%$ & 1.0 & $9.1 \%$ & 0.324 \\
\hline \multicolumn{8}{|l|}{ Medications } \\
\hline Beta-blocker & 28 & $82.3 \%$ & 19 & $82.6 \%$ & 9 & $81.8 \%$ & 0.478 \\
\hline ARB or ACEI & 26 & $76.4 \%$ & 17 & $73.9 \%$ & 9 & $81.8 \%$ & 0.312 \\
\hline Pain duration (day) & 1.0 & $0.0-2.0$ & 1.0 & $0.0-1.0$ & 2.0 & $1.5-3.5$ & 0.001 \\
\hline Pain score & 6.0 & $0.0-7.0$ & 6.0 & $0.0-7.0$ & 5.0 & $4.5-7$ & 0.197 \\
\hline Abdominal pain & 24.0 & $70.6 \%$ & 14.0 & $60.9 \%$ & 10.0 & $90.9 \%$ & 0.077 \\
\hline Nausea & 6.0 & $17.6 \%$ & 3.0 & $13.0 \%$ & 3.0 & $27.3 \%$ & 0.288 \\
\hline Vomiting & 7.0 & $20.6 \%$ & 4.0 & $17.4 \%$ & 3.0 & $27.3 \%$ & 0.404 \\
\hline \multicolumn{8}{|c|}{ CT morphology } \\
\hline SMA dissection length (mm) & 65.8 & $38.3-107.3$ & 62.0 & $22.3-87.1$ & 80.0 & $66.0-117.0$ & 0.034 \\
\hline TLD_T0 (mm) & 12.0 & $11.0-13.0$ & 12.0 & $11.0-13.0$ & 12.0 & $11.0-13.0$ & 0.240 \\
\hline TD_T0 (mm) & 5.0 & $4.0-6.0$ & 6.0 & $5.0-6.0$ & 4.0 & $2.0-5.0$ & 0.005 \\
\hline FD_T0 (mm) & 7.0 & $6.0-9.0$ & 6.0 & $5.0-8.0$ & 8.0 & $8.0-9.0$ & 0.351 \\
\hline \multicolumn{8}{|c|}{ Yun classification } \\
\hline $\mathrm{I}$ & 4.0 & $11.8 \%$ & 4.0 & $17.4 \%$ & 0.0 & $0.0 \%$ & 0.191 \\
\hline IIa & 5.0 & $14.7 \%$ & 4.0 & $17.4 \%$ & 1.0 & $9.1 \%$ & 0.471 \\
\hline $\mathrm{IIlb}$ & 23.0 & $67.6 \%$ & 14.0 & $60.9 \%$ & 9.0 & $81.8 \%$ & 0.206 \\
\hline III & 2.0 & $5.9 \%$ & 1.0 & $4.3 \%$ & 1.0 & $9.1 \%$ & 0.549 \\
\hline ICU stay (day) & 0.0 & $0.0-0.8$ & $0.0-0.5$ & $0.0-0.5$ & 0.0 & $0.0-1.0$ & 0.403 \\
\hline
\end{tabular}


Table 1. Cont.

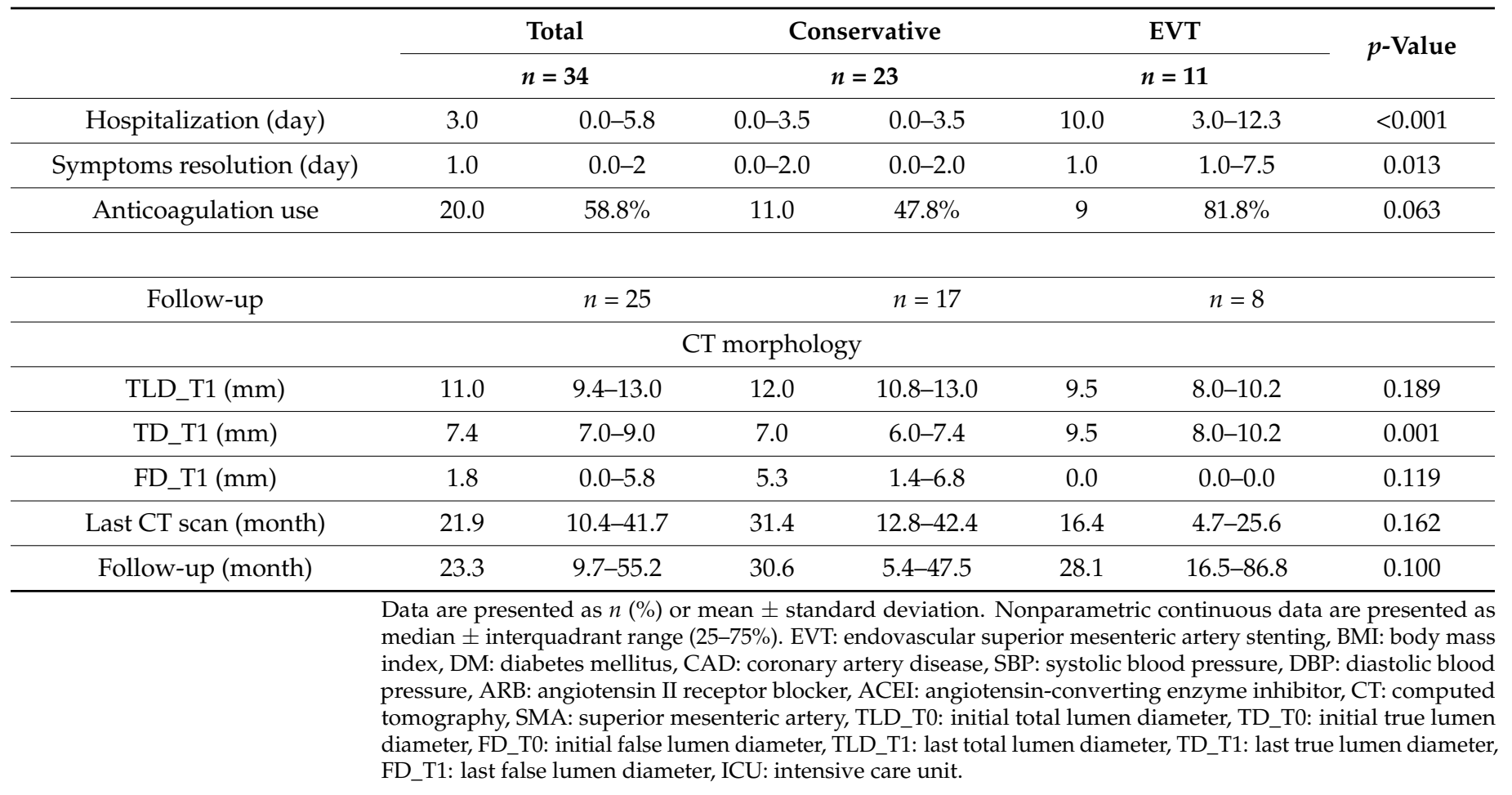

\subsection{Patients Outcomes}

In the interventional treatment, one patient required open laparotomy for SMA bypass with saphenous venous graft and bowel resection due to the mesenteric ischemia. This patient survived to discharge without sequelae. Ten $(29.4 \%)$ patients were sent to the hybrid room (Artis zeego system, Siemens Healthcare, Forchheim, Germany) for EVT. There were no peri-procedural complications. All patients experienced symptoms relief after the intervention. Clinical features and morphologic changes on CTA were available in 25 (73.5\%) patients during the mean follow-up of 23.3 months (range, 9.6-55.3 months). SMA remodeling could be observed in both conservative and EVT during the CTA follow-up at a median of 21.9 months, range:10.4-41.7. Compared to the conservative group, patients undergoing EVT had better SMA remodeling (Figure 3 and Supplementary Figure S1). After excluding asymptomatic cases, the SMA remodeling was still better in patients undergoing EVT (Supplementary Figure S2). In univariate study, SMA remodeling was significantly better in longer duration of abdominal pain, nausea, vomiting, interventional treatment, initial TL diameter, and length of SMA dissection. In the clinical outcomes, the initial presentation of abdominal pain, duration of symptom, EVT, and the length of SMA dissection were significantly related to duration of hospital stay and symptom relief. The use of anticoagulation and pain score were significantly related to the duration of symptom relief and hospital stay, respectively. However, EVT was the only significant risk factor for SMA remodeling (Table 2).

Comparing to conservative treatment, patients with Yun IIb classification were associated with better SMA remodeling after EVT (Table 3). Antithrombotic agents were not beneficial in either clinical outcomes or morphologic SMA remodeling. 
Table 2. Univariate and multivariate analysis.

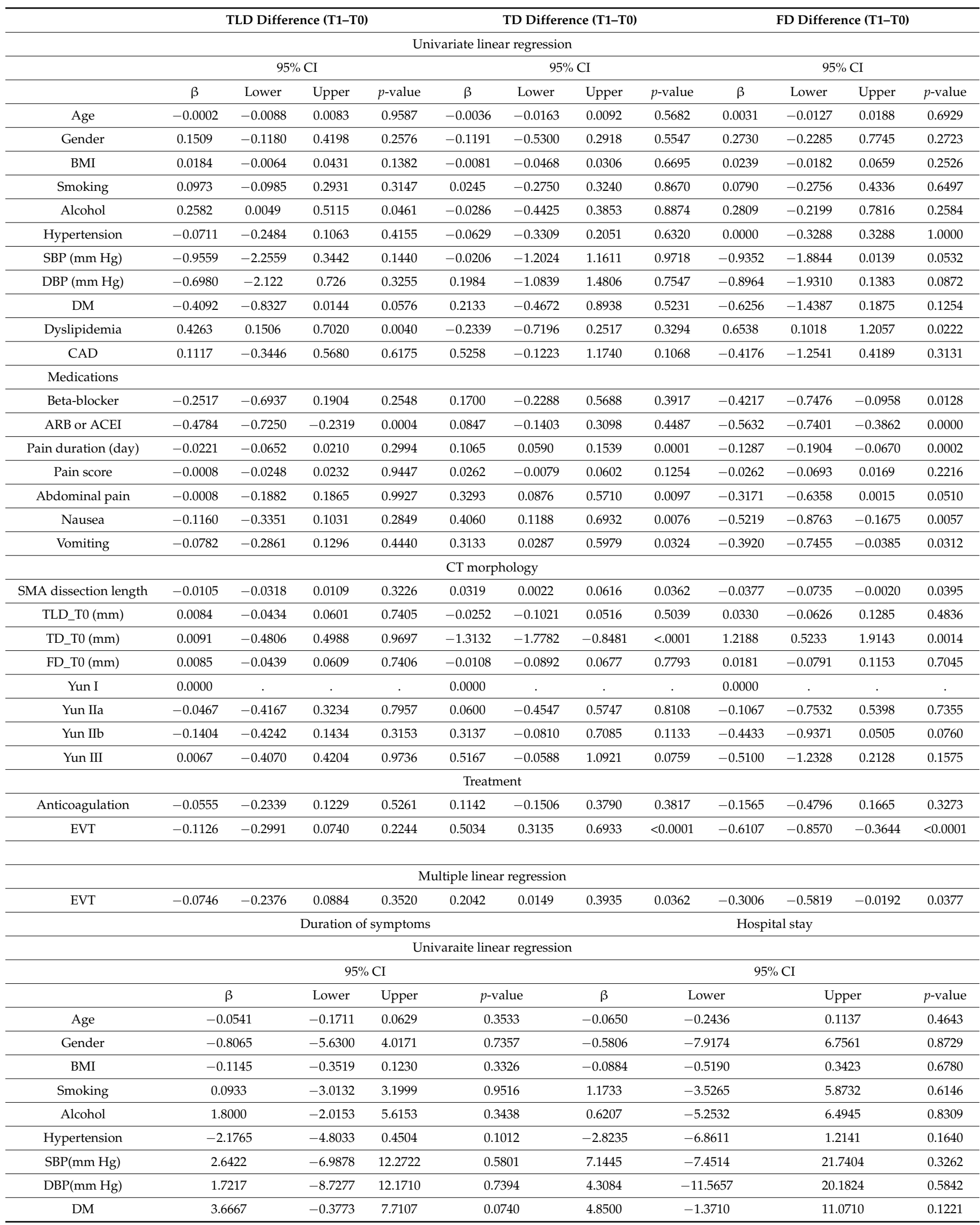


Table 2. Cont.

\begin{tabular}{|c|c|c|c|c|c|c|c|c|}
\hline \multirow[b]{2}{*}{ Dyslipidemia } & \multicolumn{3}{|c|}{ TLD Difference (T1-T0) } & \multicolumn{2}{|c|}{ TD Difference (T1-T0) } & \multicolumn{3}{|c|}{ FD Difference (T1-T0) } \\
\hline & -0.6559 & -5.4824 & 4.1706 & 0.7837 & -1.9785 & -9.2835 & 5.3266 & 0.5850 \\
\hline CAD & -0.2727 & -8.3843 & 7.8389 & 0.9458 & -2.5455 & -14.8328 & 9.7419 & 0.6759 \\
\hline \multicolumn{9}{|l|}{ Medications } \\
\hline Beta-blocker & 2.3333 & -0.9293 & 5.5960 & 0.1549 & 3.6667 & -1.2788 & 8.6121 & 0.1408 \\
\hline ARB or ACEI & 1.6842 & -0.1221 & 3.4905 & 0.0666 & 4.0000 & 1.2268 & 6.7732 & 0.0061 \\
\hline Pain duration (day) & 0.8062 & 0.1241 & 1.4883 & 0.0220 & 1.3816 & 0.3715 & 2.3916 & 0.0089 \\
\hline Pain score & 0.3061 & -0.0631 & 0.6753 & 0.1010 & 0.5685 & 0.0202 & 1.1168 & 0.0426 \\
\hline Abdominal pain & 3.2083 & 0.4309 & 5.9857 & 0.0249 & 5.7667 & 1.6969 & 9.8365 & 0.0069 \\
\hline Nausea & 0.2857 & -3.3082 & 3.8796 & 0.8724 & 2.8690 & -2.4933 & 8.2314 & 0.2839 \\
\hline Vomiting & 1.2857 & -2.0723 & 4.6437 & 0.4412 & 3.1852 & -1.8340 & 8.2044 & 0.2054 \\
\hline \multicolumn{9}{|c|}{ CT morphology } \\
\hline SMA dissection length & 0.4811 & 0.0421 & 0.9201 & 0.0327 & 0.4627 & 0.0457 & 0.8797 & 0.0306 \\
\hline TLD_T0 (mm) & -0.2562 & -1.1679 & 0.6554 & 0.5710 & -0.5638 & -1.9407 & 0.8130 & 0.4104 \\
\hline TD_T0 (mm) & -8.0605 & -14.3173 & -1.8036 & 0.0132 & -19.0484 & -26.9669 & -11.1299 & $<0.0001$ \\
\hline FD_T0 (mm) & -0.1159 & -1.0388 & 0.8070 & 0.7998 & -0.2314 & -1.6321 & 1.1693 & 0.7387 \\
\hline Yun I & 0.0000 & . & . & . & 0.0000 & . & . & ? \\
\hline Yun IIa & 0.6000 & -4.6048 & 5.8048 & 0.8155 & 1.7500 & -6.0641 & 9.5641 & 0.6507 \\
\hline Yun IIb & 3.1739 & -1.0294 & 7.3772 & 0.1335 & 5.0978 & -1.2126 & 11.4083 & 0.1094 \\
\hline Yun III & 0.5000 & -6.2194 & 7.2194 & 0.8802 & 8.7500 & -1.3379 & 18.8379 & 0.0867 \\
\hline \multicolumn{9}{|c|}{ Treatment } \\
\hline Anticoagulation & 2.7571 & 0.1552 & 5.3591 & 0.0385 & 3.3500 & -0.7044 & 7.4044 & 0.1021 \\
\hline EVT & 3.1028 & 0.3944 & 5.8112 & 0.0261 & 7.5020 & 3.9657 & 11.0383 & 0.0001 \\
\hline \multicolumn{9}{|c|}{ Multiple linear regression } \\
\hline EVT & 0.7793 & -2.3222 & 3.8807 & 0.6099 & 2.9964 & -0.1124 & 6.1052 & 0.0582 \\
\hline
\end{tabular}

$\beta$ : unadjusted regression coefficient, T0: initial computed tomography diameter, T1: last computed tomography diameter measurement, BMI: body mass index, DM: diabetes mellitus, CAD: coronary artery disease, d: day, SBP: systolic blood pressure, DBP: diastolic blood pressure, ARB: angiotensin II receptor blocker, ACEI: angiotensin-converting enzyme inhibitor, CT: computed tomography, SMA: superior mesenteric artery, TLD_T0: initial total lumen diameter, TD_T0: initial true lumen diameter, FD_T0: initial false lumen diameter, EVT: endovascular superior mesenteric artery stenting.

A
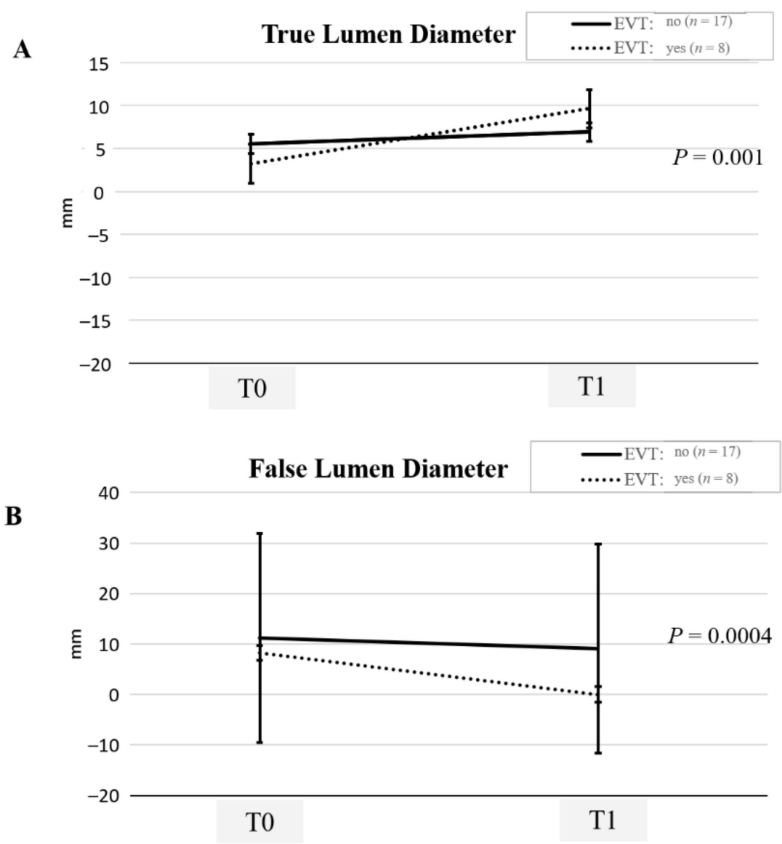

Figure 3. Superior mesenteric artery remodeling. (A) showing true lumen (TL) expansion and (B) 
false lumen (FL) reduction during the computed tomography scan follow-up. The TL expansion and FL reduction were significant better in patients undergoing superior mesenteric artery endovascular stenting (EVT).

Table 3. SMA remodeling stratified by CT morphology and EVT.

\begin{tabular}{|c|c|c|c|c|c|c|c|c|c|c|}
\hline \multirow[b]{2}{*}{$\begin{array}{c}\text { SMA } \\
\text { Remodeling }\end{array}$} & \multirow[b]{2}{*}{$\begin{array}{c}\text { CT } \\
\text { Morphology }\end{array}$} & \multirow[b]{2}{*}{$N=17$} & \multicolumn{3}{|c|}{ Conservative } & \multirow[b]{2}{*}{$N=8$} & \multicolumn{3}{|c|}{ EVT } & \multirow[b]{2}{*}{$p$-Value } \\
\hline & & & Median & IQR & (Q25-Q75) & & Median & IQR & (Q25-Q75) & \\
\hline \multirow[t]{4}{*}{$\begin{array}{l}\text { TLD difference } \\
\text { (T1-T0) }\end{array}$} & Yun I & 3 & 0.000 & 0.220 & $(-0.120-0.100)$ & 0 & NA & NA & NA & \\
\hline & Yun IIa & 3 & -0.020 & 0.260 & $(-0.200-0.060)$ & 0 & NA & NA & NA & \\
\hline & Yun IIb & 10 & -0.055 & 0.400 & $(-0.400-0.000)$ & 7 & -0.170 & 0.300 & $(-0.400-0.100)$ & 0.3671 \\
\hline & Yun III & 1 & 0.000 & 0.000 & $(0.000-0.000)$ & 1 & NA & NA & NA & \\
\hline \multirow[t]{4}{*}{$\begin{array}{l}\text { TD difference } \\
\text { (T1-T0) }\end{array}$} & Yun I & 3 & 0.000 & 0.100 & $(0.000-0.100)$ & 0 & NA & NA & NA & \\
\hline & Yun IIa & 3 & 0.100 & 0.180 & $(0.000-0.180)$ & 0 & NA & NA & NA & \\
\hline & Yun IIb & 10 & 0.140 & 0.150 & $(0.100-0.250)$ & 7 & 0.700 & 0.400 & $(0.400-0.800)$ & 0.0266 \\
\hline & Yun III & 1 & 0.100 & 0.000 & $(0.100-0.100)$ & 1 & NA & NA & NA & \\
\hline \multirow[t]{4}{*}{$\begin{array}{c}\text { FD difference } \\
(\mathrm{T} 1-\mathrm{T} 0)\end{array}$} & Yun I & 3 & -0.100 & 0.220 & $(-0.120-0.100)$ & 0 & NA & NA & NA & \\
\hline & Yun IIa & 3 & -0.200 & 0.360 & $(-0.300-0.060)$ & 0 & NA & NA & NA & \\
\hline & Yun IIb & 10 & -0.300 & 0.700 & $(-0.560-0.140)$ & 7 & -0.800 & 0.100 & $(-0.800-0.700)$ & 0.0120 \\
\hline & Yun III & 1 & -0.100 & 0.000 & $(-0.100-0.100)$ & 1 & -1.000 & 0.000 & $(-1.000-1.000)$ & \\
\hline
\end{tabular}

At a median follow-up of 23.3 months (range: 9.6-55.2), no patients developed SMA aneurysm formation or recurrent symptoms requiring admission in these two groups. There was no stent occlusion or new dissection. The long-term survival was $96 \%$. One patient died after 4 years follow-up in conservative treatment group unrelated to the SMA rupture or mesenteric ischemia.

\section{Discussion}

This study showed that SMA stenting in patients with SIDSMA was significantly better for SMA remodeling compared to medical treatment, especially in patients presenting with Yuan's type IIb. After a median 23.3 months follow-up, no SMA aneurysm or deaths related to mesenteric ischemia were noted after either conservative and endovascular treatment.

SISMAD represented the dominant types of visceral artery dissection [11,14], and the incidence was reported to be approximately $0.06-0.08 \%[1,9,11]$. In the current guideline, treatment of SIDSMA is aimed at controlling symptoms and preventing complications (e.g., bowel necrosis, aneurysm rupture) [9]. Most studies suggested initial treatment according to the clinical presentation on admission. If SIDSMA was identified as an incidental finding on CTA performed for other conditions, the patient could be managed by careful observation and conservative management $[18,19]$. None of the asymptomatic patients treated conservatively required secondary intervention [11]. However, in symptomatic patients, the failure rate ranged from $5.0-44.0 \%$ depending on the different reports [14]. In our study, $45.8 \%$ of symptomatic patients $(11 / 24)$ had persistent abdominal pain and received the interventional therapy. One reason for this higher rate of intervention might be the threshold of the duration for conservative treatment. Currently there is no clear definition of the duration for the symptom relief indicating the failure of conservative treatment. The duration could range from a few days to weeks depending on the existing, or development of, collaterals $[1,9,16]$. Usually, conservative treatment would take longer 
to achieve symptom relief compared to surgery or EVT [16]. In our study, the average waiting period was two days after the initial abdominal pain to guide the interventional treatment option. The concern for bowel ischemia on clinical or radiologic examination was considered an indication for early intervention. Pain from the dissection itself was often confused with the presence of bowel ischemia and made the clinical evaluation difficult. Other laboratory markers such as leukocytosis, lactate, and amylase were neither sensitive nor specific. If any secondary signs of intestinal mal-perfusion, including bowel-wall thickening, pneumatosis, or lack of mucosal enhancement, appeared in the follow-up CTA, usually the golden period for the EVT had elapsed. The addition of antithrombotic agents did not prevent the progression of dissection and facilitate the development of collateral vessels in a short period of time $[1,9,11,15,16,19]$, as shown in this study, where the antithrombotic agents did not prevent patients undergoing intervention treatment from receiving conservative treatment.

Given the weakening of the dissected SMA arterial wall, one might expect aneurysmal degeneration or recurrent dissection in the long-term follow-up, and the patients with favorable SMA remodeling overtime could be a surrogate for the long-term remission of the SIDSMA [9]. Currently, there is a uniform definition but only qualitative description regarding the SMA remodeling. Most studies compared the SMA remodeling as either complete or incomplete classifications. Complete remodeling was defined as SMA remodeling with no evidence of abnormality, and incomplete remodeling as increase in TL size with or without reduction in FL size in the follow-up CTA [15-17]. In the literature, the reported complete or incomplete remodeling rate after conservative treatment was quite variable; reported as $63.0 \%$ of patients at a mean follow-up of $16 \pm 16$ months after initial pain relief in the study from Heo et al. [16] and 12.6\% in the study from Qiu et al. [17]. However, the reported rate of recurrent abdominal pain was quite similar in both studies (20.0-25.0\%) [1,5,15-17]. Based on the reporting standard for type B aortic dissections, positive aortic remodeling should be classified as TL expansion or FL reduction with or without reduction of total aortic diameter [20]. In this study, we adopted the principle to define the SMA remodeling based on the TL, FL, and total arterial diameter change and compared between the EVT and conservative treatment. In our study, patients undergoing EVT achieved significantly better SMA remodeling (quantitative measurement, instead of qualitative description only) compared to patients receiving conservative treatment. In our study, we could not appreciate the importance of SMA remodeling in the recurrent abdominal pain because no recurrent symptoms were noted in both groups. It seemed that the recurrent abdominal pain was not really related to the SMA remodeling but more related to the collateral formation from the celiac or IMA axis after the dissection event.

Regarding the morphologic difference, symptomatic patients usually had longer length of SMA dissection than asymptomatic patients did [11].The average length of dissection ranged from $6.5 \mathrm{~cm}$ to $9.5 \mathrm{~cm}[15,17]$. In our study, the median length of SMA dissection was $65.8 \mathrm{~mm}$, and a significant difference was found between interventional and conservative treatment groups. More than six morphological classifications of SIDSMA have been proposed $[1,5,9,16,21]$. All were based on imaging appearance of the SIDSMA, such as the extent of the FL, entry and/or reentry visible, and presence of thrombosis in the FL and/or TL. However, none of these classifications could predict the clinical course. In our study, patients with Yun's IIb classification CT morphology had significant better SMA remodeling in CTA follow-up in the treatment group, but no difference in midterm outcomes compared to patients receiving the medical treatment. This represented that patient who were symptomatic with dismal morphological appearance could have same midterm outcome after EVT treatment.

In the literature, the range of hospital stay was around 7 days in the conservative treatment group. In our study, comparing to patients under conservative treatment, patients undergoing interventional therapy had longer hospital stay because of the prolonged initial conservative treatment and the post-procedure recovery stay. For those symptomatic patients who received conservative treatment initially, secondary intervention was required 
at an estimated $12.0 \%$ of the SIDSMA patients during the follow-up [11]. However, in our study, no readmission for secondary intervention was related to the adverse events from SMA, including recurrent abdominal pain, aneurysmal formation, or new dissection. There was only one case requiring open SMA bypass surgery with saphenous vein graft from right common iliac artery and segmental bowel resection in early 2007. No other open surgical case was performed afterward. It may have been due to early diagnosis by CTA and early intervention management for prolonged abdominal pain before bowel necrosis happened. There was no EVT failure requiring conversion to open surgery in this study. The long-term survival was $96 \%$ at the median follow-up of 23.3 months. One patient died after 4 years follow-up in conservative treatment group unrelated to the SMA rupture or mesenteric ischemia. The long-term survival was compatible to previous reports explaining that rarely was the cause of mortality related to SMA rupture or ischemia $[5,15,16]$.

This study has some limitations. First, it is a retrospective study in a single institution with a limited number of patients due to the rarity of SIDSMA. The assessment of sample size from previous studies and statistical power was not available due to the variations of the treatment modality $[16,17]$. As a result of our small sample size, multivariable analysis was not possible in some variables. The result might not be generalizable. However, it still remained the first study in the literature with the quantitative measurement of the SMA remodeling. Second, several patients did not have follow-up CTA images available in our electronic record system, and the follow-up imaging and timing were not uniform. In the CT scan, we could not differentiate the quantitative collateral circulations between symptomatic and asymptomatic patients.

\section{Conclusions}

In this study, SIDSMA could be initially managed by medical treatment. In symptomatic SIDSMA, EVT could be performed if the symptom persisted and before the mesenteric ischemia progressed. SMA remodeling was significantly better after EVT, especially with Yun's IIb classification morphology. Anticoagulation therapy had no impact on the natural history of the SIDSMA. Secondary intervention was rarely required after both conservative and interventional treatments.

Supplementary Materials: The following supporting information can be downloaded at: https: / / www.mdpi.com/article/10.3390/jcm11020465/s1, Figure S1: Total superior mesenteric artery diameter change during the computed tomography scan follow up; Figure S2: Total, true and false lumen of superior mesenteric artery diameter change during the computed tomography scan follow up after excluding asymptomatic cases; Table S1: Patients demographic, CT scan morphology and follow-up data.

Author Contributions: Conceptualization: S.-H.Y., I.-H.W., data curation: S.-H.Y., I.-H.H., I.-H.W. formal analysis: S.-H.Y., I.-H.H., I.-H.W., investigation: S.-H.Y., I.-H.H., I.-H.W., methodology: S.-H.Y., I.-H.H., I.-H.W., project administration: I.-H.W., resources: I.-H.W., software: S.-H.Y., I.-H.H., I.-H.W., supervision: I.-H.W., validation: S.-H.Y., I.-H.H., I.-H.W., visualization: S.-H.Y., I.-H.H., I.-H.W., writing - original draft: S.-H.Y., I.-H.H., writing—review and editing: I.-HW. All authors have read and agreed to the published version of the manuscript.

Funding: This research received no external funding.

Institutional Review Board Statement: This study was approved by the institutional review board of National Taiwan University Hospital (NTUH-202104075RINA), and the board waived the need for informed consent.

Informed Consent Statement: Patient consent was waived due to the retrospective nature.

Data Availability Statement: Not applicable.

Acknowledgments: The authors would like to express their thanks to the staff of National Taiwan University Hospital-Statistical Consulting Unit (NTUH-SCU) for statistical consultation and analyses.

Conflicts of Interest: The authors declare no conflict of interest. 


\section{References}

1. Luan, J.Y.; Guan, X.; Li, X.; Wang, C.M.; Li, T.R.; Zhang, L.; Han, J.T. Isolated superior mesenteric artery dissection in China. J. Vasc. Surg. 2016, 63, 530-536. [CrossRef]

2. Park, Y.J.; Park, K.B.; Kim, D.I.; Do, Y.S.; Kim, D.K.; Kim, Y.W. Natural history of spontaneous isolated superior mesenteric artery dissection derived from follow-up after conservative treatment. J. Vasc. Surg. 2011, 54, 1727-1733. [CrossRef] [PubMed]

3. Yun, W.S.; Kim, Y.W.; Park, K.B.; Cho, S.K.; Do, Y.S.; Lee, K.B.; Kim, D.I.; Kim, D.K. Clinical and angiographic follow-up of spontaneous isolated superior mesenteric artery dissection. Eur. J. Vasc. Endovasc. Surg. 2009, 37, 572-577. [CrossRef] [PubMed]

4. Tomita, K.; Obara, H.; Sekimoto, Y.; Matsubara, K.; Watada, S.; Fujimura, N.; Shibutani, S.; Nagasaki, K.; Hayashi, S.; Harada, H.; et al. Evolution of Computed Tomographic Characteristics of Spontaneous Isolated Superior Mesenteric Artery Dissection During Conservative Management. Circ. J. 2016, 80, 1452-1459. [CrossRef]

5. $\quad$ Li, D.L.; He, Y.Y.; Alkalei, A.M.; Chen, X.D.; Jin, W.; Li, M.; Zhang, H.K.; Liang, T.B. Management strategy for spontaneous isolated dissection of the superior mesenteric artery based on morphologic classification. J. Vasc. Surg. 2014, 59, 165-172. [CrossRef] [PubMed]

6. Kim, H.K.; Jung, H.K.; Cho, J.; Lee, J.M.; Huh, S. Clinical and radiologic course of symptomatic spontaneous isolated dissection of the superior mesenteric artery treated with conservative management. J. Vasc. Surg. 2014, 59, 465-472. [CrossRef] [PubMed]

7. Jia, Z.Z.; Zhao, J.W.; Tian, F.; Li, S.Q.; Wang, K.; Wang, Y.; Jiang, L.Q.; Jiang, G.M. Initial and middle-term results of treatment for symptomatic spontaneous isolated dissection of superior mesenteric artery. Eur. J. Vasc. Endovasc. Surg. 2013, 45, 502-508. [CrossRef] [PubMed]

8. Cho, B.S.; Lee, M.S.; Lee, M.K.; Choi, Y.J.; Kim, C.N.; Kang, Y.J.; Park, J.S.; Ahn, H.Y. Treatment guidelines for isolated dissection of the superior mesenteric artery based on follow-up CT findings. Eur. J. Vasc. Endovasc. Surg. 2011, 41, 780-785. [CrossRef] [PubMed]

9. Bjorck, M.; Koelemay, M.; Acosta, S.; Bastos Goncalves, F.; Kolbel, T.; Kolkman, J.J.; Lees, T.; Lefevre, J.H.; Menyhei, G.; Oderich, G.; et al. Editor's Choice-Management of the Diseases of Mesenteric Arteries and Veins: Clinical Practice Guidelines of the European Society of Vascular Surgery (ESVS). Eur. J. Vasc. Endovasc. Surg. 2017, 53, 460-510. [CrossRef]

10. Sakamoto, I.; Ogawa, Y.; Sueyoshi, E.; Fukui, K.; Murakami, T.; Uetani, M. Imaging appearances and management of isolated spontaneous dissection of the superior mesenteric artery. Eur. J. Radiol. 2007, 64, 103-110. [CrossRef]

11. Wang, J.; He, Y.; Zhao, J.; Yuan, D.; Xu, H.; Ma, Y.; Huang, B.; Yang, Y.; Bian, H.; Wang, Z. Systematic review and meta-analysis of current evidence in spontaneous isolated celiac and superior mesenteric artery dissection. J. Vasc. Surg. 2018, 68, 1228-1240.e9. [CrossRef]

12. Garrett, H.E., Jr. Options for treatment of spontaneous mesenteric artery dissection. J. Vasc. Surg. 2014, 59, 1433-1439.e2. [CrossRef] [PubMed]

13. Kimura, Y.; Kato, T.; Inoko, M. Outcomes of Treatment Strategies for Isolated Spontaneous Dissection of the Superior Mesenteric Artery: A Systematic Review. Ann. Vasc. Surg. 2018, 47, 284-290. [CrossRef]

14. Zettervall, S.L.; Karthaus, E.G.; Soden, P.A.; Buck, D.B.; Ultee, K.H.; Schermerhorn, M.L.; Wyers, M.C. Clinical presentation, management, follow-up, and outcomes of isolated celiac and superior mesenteric artery dissections. J. Vasc. Surg. 2017, 65, 91-98. [CrossRef] [PubMed]

15. Kim, H.; Park, H.; Park, S.J.; Park, B.W.; Hwang, J.C.; Seo, Y.W.; Cho, H.R. Outcomes of Spontaneous Isolated Superior Mesenteric Artery Dissection Without Antithrombotic Use. Eur. J. Vasc. Endovasc. Surg. 2018, 55, 132-137. [CrossRef] [PubMed]

16. Heo, S.H.; Kim, Y.W.; Woo, S.Y.; Park, Y.J.; Park, K.B.; Kim, D.K. Treatment strategy based on the natural course for patients with spontaneous isolated superior mesenteric artery dissection. J. Vasc. Surg. 2017, 65, 1142-1151. [CrossRef] [PubMed]

17. Qiu, C.; He, Y.; Li, D.; Shang, T.; Wang, X.; Wu, Z.; Zhang, H. Mid-Term Results of Endovascular Treatment for Spontaneous Isolated Dissection of the Superior Mesenteric Artery. Eur. J. Vasc. Endovasc. Surg. 2019, 58, 88-95. [CrossRef]

18. Kimura, Y.; Kato, T.; Nagao, K.; Izumi, T.; Haruna, T.; Ueyama, K.; Inada, T.; Inoko, M. Outcomes and Radiographic Findings of Isolated Spontaneous Superior Mesenteric Artery Dissection. Eur. J. Vasc. Endovasc. Surg. 2017, 53, 276-281. [CrossRef]

19. Loeffler, J.W.; Obara, H.; Fujimura, N.; Bove, P.; Newton, D.H.; Zettervall, S.L.; van Petersen, A.S.; Geelkerken, R.H.; CharltonOuw, K.M.; Shalhub, S.; et al. Medical therapy and intervention do not improve uncomplicated isolated mesenteric artery dissection outcomes over observation alone. J. Vasc. Surg. 2017, 66, 202-208. [CrossRef]

20. Lombardi, J.V.; Hughes, G.C.; Appoo, J.J.; Bavaria, J.E.; Beck, A.W.; Cambria, R.P.; Charlton-Ouw, K.; Eslami, M.H.; Kim, K.M.; Leshnower, B.G.; et al. Society for Vascular Surgery (SVS) and Society of Thoracic Surgeons (STS) reporting standards for type B aortic dissections. J. Vasc. Surg. 2020, 71, 723-747. [CrossRef]

21. Zerbib, P.; Perot, C.; Lambert, M.; Seblini, M.; Pruvot, F.R.; Chambon, J.P. Management of isolated spontaneous dissection of superior mesenteric artery. Langenbecks Arch. Surg. 2010, 395, 437-443. [CrossRef] [PubMed] 